\title{
Macromolecular Crowding Enhances the Detection of DNA and Proteins by a Solid-State Nanopore
}

\author{
Chalmers C. Chau, Sheena E. Radford, Eric W. Hewitt,* and Paolo Actis* \\ Cite This: Nano Lett. 2020, 20, 5553-5561 \\ Read Online
}

ABSTRACT: Nanopore analysis of nucleic acid is now routine, but detection of proteins remains challenging. Here, we report the systematic characterization of the effect of macromolecular crowding on the detection sensitivity of a solid-state nanopore for circular and linearized DNA plasmids, globular proteins ( $\beta$-galactosidase), and filamentous proteins ( $\alpha$-synuclein amyloid fibrils). We observe a remarkable ca. 1000 -fold increase in the molecule count for the globular protein $\beta$-galactosidase and a 6 -fold increase in peak amplitude for plasmid DNA under crowded conditions. We also demonstrate that macromolecular crowding facilitates the study of the topology of DNA plasmids and the characterization of amyloid fibril preparations with different length distributions. A remarkable feature of this method is its ease of use; it

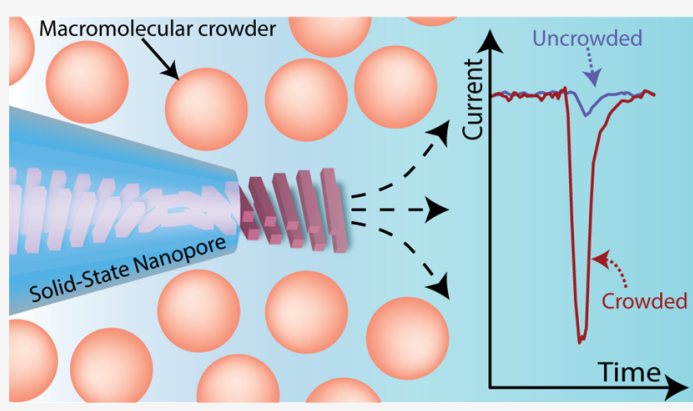
simply requires the addition of a macromolecular crowding agent to the electrolyte. We therefore envision that macromolecular crowding can be applied to many applications in the analysis of biomolecules by solid-state nanopores.

KEYWORDS: Nanopore, nanopipette, macromolecular crowding, single-molecule, DNA, protein, amyloid fibril

Tn the last 20 years, nanopore sensing has emerged as a key enabling technology for biomolecular analysis at the single molecule level. ${ }^{1,2}$ Recent advancements enabled the development of a nanopore-based platform for DNA and RNA sequencing, ${ }^{3-5}$ but protein analysis remains challenging. Plesa et al. demonstrated that the bulk of proteins translocate on time scales faster than the tens of microseconds detection limit of commonly used patch-clamp amplifiers, and they proposed two solutions to improve the efficiency of the detection. ${ }^{6}$ The first solution involves slowing down the speed at which proteins translocate through the nanopores which can be achieved by careful selection of electrolytes, ${ }^{7-9}$ chemical and biological modification of nanopores, ${ }^{10-13}$ or the use of nucleic-acid-based carrier molecules. ${ }^{14,15}$ The second solution involves the development of high bandwidth electronics to improve the amplifier temporal resolution. ${ }^{16-18}$

Herein, we demonstrate the use of a macromolecular crowded electrolyte bath as the third approach to increase the sensitivity of a solid-state nanopore and improve its detection efficiency. We demonstrate that the macromolecular crowder polyethylene glycol (PEG) $8000^{19}$ dramatically enhances the detection of DNA and proteins in a solid-state nanopore both in terms of molecule count and peak amplitude, with a near 1000-fold increase in the molecule count for the globular protein $\beta$-galactosidase (Figure 1). Furthermore, macromolecular crowding enabled the characterization of filamentous proteins ( $\alpha$-synuclein amyloid fibrils) which were hard to detect under noncrowded conditions. We also demonstrate that macromolecular crowding facilitates the study of the topology of DNA plasmids and the character- ization of amyloid fibril preparations with different length distributions.

Solid-state nanopores were fabricated by laser pulling quartz capillaries, resulting in nanopipettes with nanopores at their tips $10-15 \mathrm{~nm}$ in diameter based on scanning electron microscopy imaging and resistance estimation (Supporting Figure $1 \mathrm{~A}){ }^{20,21}$ The nanopipette was filled with $0.01 \%(\mathrm{w} / \mathrm{v})$ Tween-20 in phosphate buffered saline (PBS) and fitted with an $\mathrm{Ag} / \mathrm{AgCl}$ electrode. Commonly, the salt of choice for single molecule detection with nanopores is $\mathrm{KCl}, \mathrm{NaCl}$, or $\mathrm{LiCl}$ to improve the signal-to-noise ratio. ${ }^{9}$ Here, we used PBS, a commonly used physiological buffer, as the electrolyte. $\alpha$ Synuclein amyloid fibrils were assembled in PBS, and thus for consistency, this buffer was used for the detection of all the biomolecules in this study. The nanopipette and an $\mathrm{Ag} / \mathrm{AgCl}$ reference electrode were then immersed into the PBS electrolyte solution containing either different concentrations of the viscogen glycerol or the crowding agent PEG 8000. The ionic strength of the bath was kept constant in all experiments to eliminate any osmotic effects. ${ }^{8}$ The nanopipette used in this study showed a negative ion current rectification which was reversed by the presence of glycerol or PEG 8000 in the bath

Received: May 28, 2020

Revised: June 18, 2020

Published: June 19, 2020 


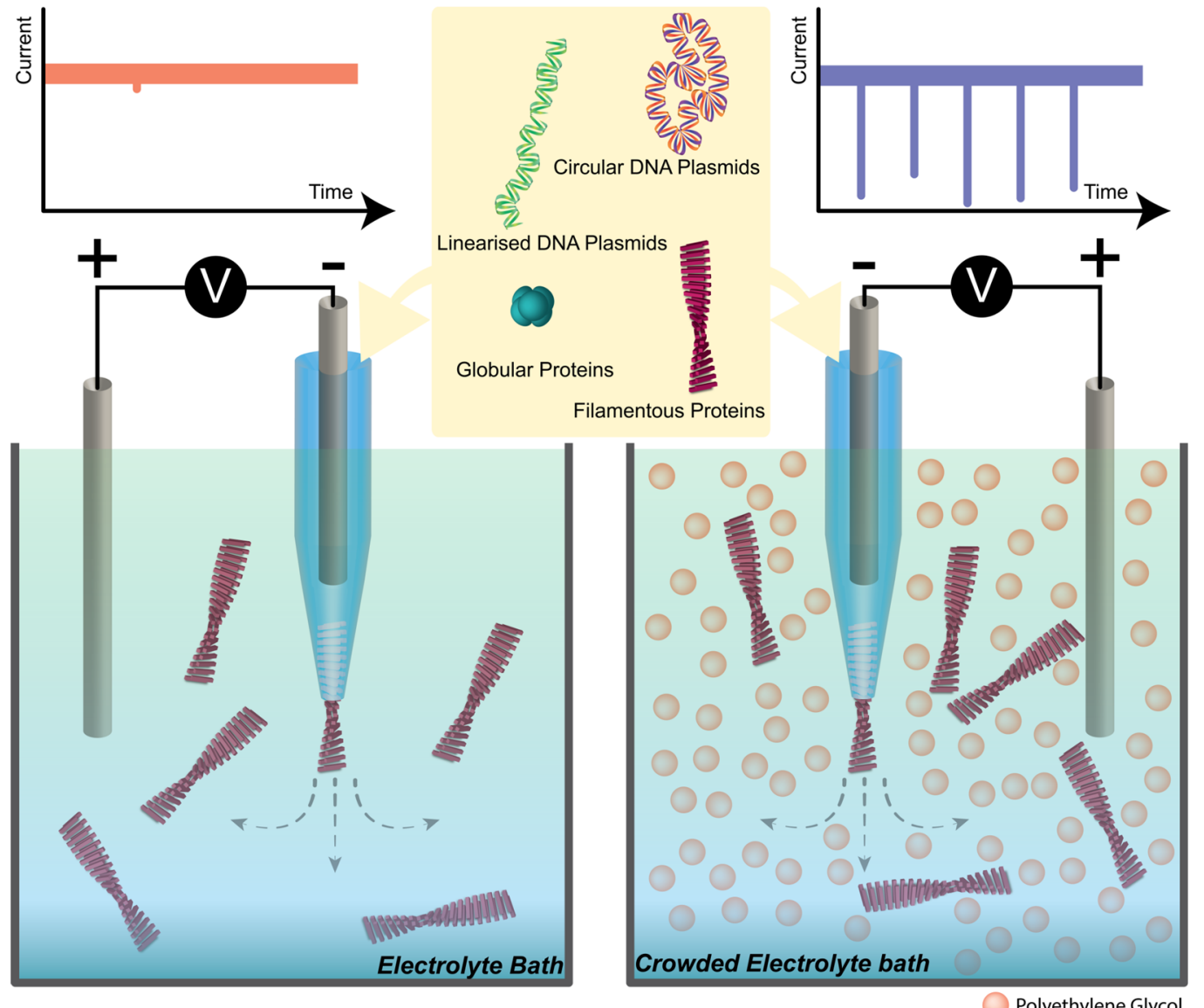

Figure 1. Macromolecular crowding increases the sensitivity for detection of macromolecules by nanopore sensing.

with the most marked reversal observed in $50 \%(\mathrm{w} / \mathrm{v})$ PEG 8000 (Supporting Figure 1B-D). These results have been previously observed by coating the nanopipette with positively charged polyelectrolytes, such as poly-L-lysine ${ }^{22,23}$ and are consistent with a modification of the electroosmotic flow within the nanopore, ${ }^{23,24}$ suggesting that both glycerol and PEG 8000 affect the nanofluidic properties of the nanopore in a similar way. The positive ion current rectification observed in $50 \%(\mathrm{w} / \mathrm{v})$ PEG 8000 could be due to the cation binding properties of PEG. Studies have shown that the flexible polyether chain is able to capture and intercalate cations into the molecule structure and to affect the ion current rectification. $^{25-27}$

We then studied the translocation dynamics of a $3.5 \mathrm{kbp}$ circular DNA plasmid (see the Supporting Information) to investigate the effect of crowding and viscosity on a wellcharacterized biomolecule. A nanopipette was filled with PBS containing $0.01 \%(\mathrm{w} / \mathrm{v})$ Tween-20 and $1.33 \mathrm{pM}$ of the circular DNA plasmid. Different voltages were tested for the translocation of the circular DNA plasmid (Supporting Figure 2). For all DNA plasmid translocation experiments hereinafter, a potential difference of $-700 \mathrm{mV}$ was used and applied to the electrode inside the nanopipette for $60 \mathrm{~s}$ to drive electrophoretically the translocation of the biomolecules out of the nanopipette and into the electrolyte bath (Figure 2, Supporting Figure 3). The translocation of DNA through a nanopipette induces a temporary increase in the conductance rather than a decrease, and this effect has been observed before both in nanopipettes and other solid-state nanopores. ${ }^{28-30}$ This effect is due to charged counterions shielding the negatively charged
DNA molecule and differences in the effects of ion concentration modulation and geometrical exclusion of ions when the DNA molecules approached the nanopore. ${ }^{30-32}$

In PBS, the translocation events had an average molecule count of $360 \pm 5$ per $60 \mathrm{~s}$ of recording (Figures $2 \mathrm{~A}$ and $2 \mathrm{D}$ ), with an average peak amplitude of $88 \pm 1 \mathrm{pA}$ (Figure $2 \mathrm{E}$ ) and an average dwell time of $111 \pm 10 \mu \mathrm{s}$ (Figure $2 \mathrm{~F}$ ). Increasing glycerol concentrations to $12.5 \%(\mathrm{v} / \mathrm{v})$ or $25 \%(\mathrm{v} / \mathrm{v})$ caused a decrease in molecule count leading to $67 \pm 23$ molecules per $60 \mathrm{~s}$ at $50 \%(\mathrm{v} / \mathrm{v})$ glycerol (Figures $2 \mathrm{~B}$ and $2 \mathrm{D})$ ). Furthermore, the viscous glycerol electrolyte bath had a negligible effect on the average current amplitude and dwell time regardless of the concentration except at $12.5 \%$ where the dwell time is statistically significantly lower than the PBS (Figures $2 \mathrm{E}$ and $2 \mathrm{~F}$ ). In contrast, measurements performed in PBS containing increasing concentrations of PEG 8000 showed a pronounced increase in the molecule count $(743 \pm 36$ in $12.5 \%, 1308 \pm$ 156 in $25 \%$, and $1063 \pm 24$ molecules per $60 \mathrm{~s}$ in $50 \%(\mathrm{w} / \mathrm{v})$ PEG 8000 (Figures 2C and 2D). Interestingly, we observed a 6-fold increase in average peak amplitude in 50\% (w/v) PEG 8000 , from $88 \pm 1 \mathrm{pA}$ in PBS buffer alone to $496 \pm 8 \mathrm{pA}$ in $50 \%(\mathrm{w} / \mathrm{v}$ ) PEG 8000 (Figures $2 \mathrm{E}$ and $2 \mathrm{~F}$ ). On the other hand, the average dwell time of $127 \pm 10 \mu \mathrm{s}$ in $50 \%(\mathrm{w} / \mathrm{v})$ PEG 8000 did not show a statistically significant difference to that observed in PBS alone $(111 \pm 10 \mu \mathrm{s}$ ) (Figure 2F), while lower concentrations of PEG 8000 caused the events to have a statistically significant shorter dwell time $(80 \pm 10$ and $72 \pm 10$ $\mu$ s for $12.5 \%(\mathrm{w} / \mathrm{v})$ and $25 \%(\mathrm{w} / \mathrm{v})$, respectively) (Figure $2 \mathrm{~F}$ ). The effect of $50 \%(\mathrm{w} / \mathrm{v})$ PEG 8000 on the translocation dynamics of the DNA plasmid is instantly reversible, indicating 

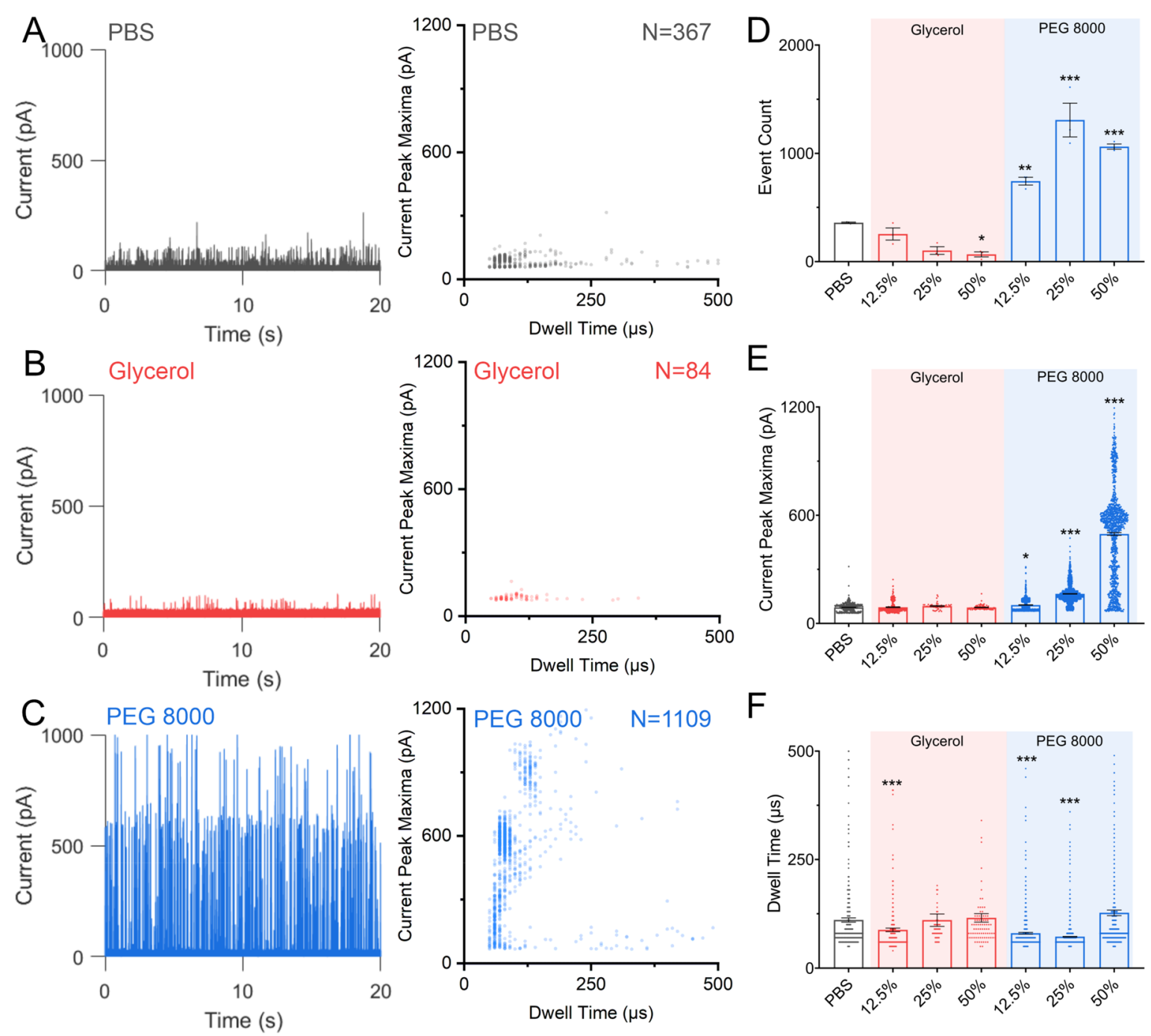

Figure 2. Macromolecular crowding enhances the detection of plasmid DNA. The baseline subtracted current trace for the first $20 \mathrm{~s}$ is shown for electrolyte baths of either (A) PBS, (B) PBS with 50\% (v/v) glycerol, or (C) PBS with 50\% (w/v) PEG 8000. The scatter plot shows the dwell time versus the current peak maxima for each event for the entire $60 \mathrm{~s}$ recording, $N$ refers to the total number of recorded events. The presence of PEG 8000 in the electrolyte bath resulted in a pronounced increase in the (D) event count, (E) current peak maxima, and (F) dwell time of events when compared to PBS. Asterisks show where the addition of either glycerol or PEG 8000 resulted in a statistically significant change when compared to PBS alone or PBS and glycerol. Error bars are \pm SEM. Asterisks indicate $P$-values $(* P<0.5, * * P<0.1, * * * P<0.01)$. One-way ANOVA test was used for (D), and the Kruskal-Wallis test was used for (E) and (F). The data points in bar charts (E and F) correspond to individual events from the current traces. See also Supporting Figure 3.

that the effect is caused by macromolecular crowding in solution rather than PEG 8000 adsorption onto the nanopore walls (Supporting Figure 4). Nanopores have been used to detect PEG 8000 molecules. ${ }^{33,34}$ Therefore, to ensure that the detected events were indeed due to the DNA plasmid, a control nanopipette without added plasmid was immersed into $50 \%(\mathrm{w} / \mathrm{v})$ PEG 8000 bath, and no single molecule events were detected (Supporting Figure 5).

Circular DNA plasmids exist in both relaxed and supercoiled states and can be linearized by digestion with restriction enzymes. ${ }^{35}$ Building on observations that nanopores can distinguish the different topological states of a plasmid and even more complex DNA knots, we also studied the effect of macromolecular crowding on the analysis of circular and linearized plasmid DNA. ${ }^{36-38}$ The $3.5 \mathrm{kbp}$ circular plasmid was linearized by digestion with the restriction enzyme KpnI, as confirmed by gel electrophoresis (Supporting Figure 6). A nanopipette was filled with $1.33 \mathrm{pM}$ of either the circular or linearized DNA plasmid, and translocation experiments were carried out in either 50\% (w/v) PEG 8000 in PBS or PBS alone. Differences between current amplitudes for the circular and linearized plasmid DNAs were observed in both electrolyte solutions, but these differences were more pronounced in the presence of $50 \%(\mathrm{w} / \mathrm{v})$ PEG 8000 (Figures $3 \mathrm{~A}$ and 3B, Supporting Figure 7C). In 50\% (w/v) PEG 8000 the majority of events for the linearized plasmid were below $600 \mathrm{pA}$, with an average current amplitude of $287 \pm 4 \mathrm{pA}$ (Figure 3C). In marked contrast for the circular plasmid there was not only a statistically significant increase in average current amplitude $(544 \pm 7 \mathrm{pA})$ but there was also a distinct population of events at $\sim 900 \mathrm{pA}$ that were not observed for the linearized plasmid (Figures 3A and 3B). This more complex distribution of events for the circular plasmid may reflect the presence of both relaxed and supercoiled forms of circular DNA. Moreover, there was also a statistically significant increase in average dwell 

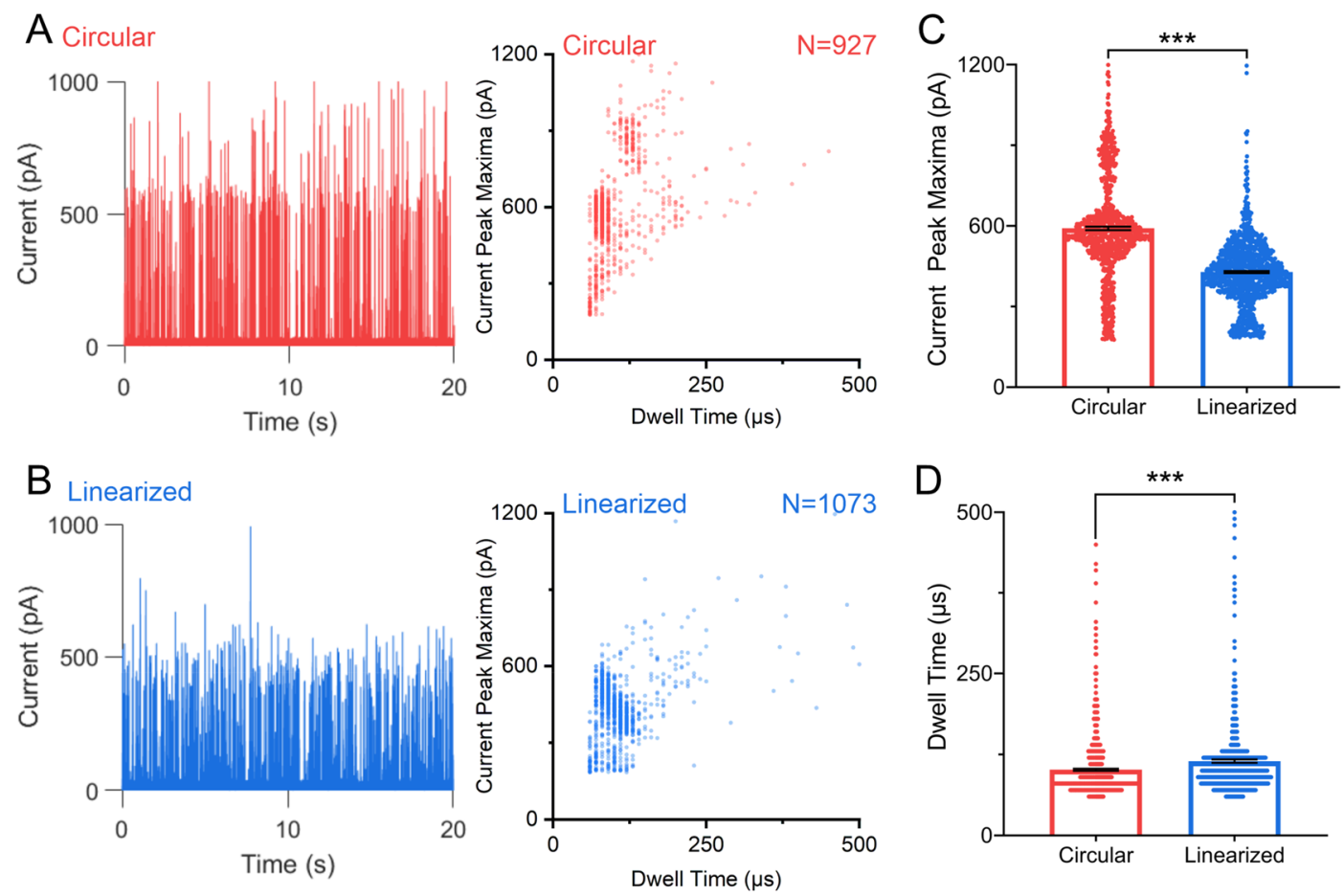

Figure 3. Macromolecular crowding enables the detection of plasmid DNA topologies. Baseline subtracted current trace for the first $20 \mathrm{~s}$ is shown for (A) circular or (B) linearized plasmid DNA in 50\% (w/v) PEG 8000, with a scatter plot to show the dwell time as a function of the current peak maxima for each event. Note that circular DNA has a distinctive population of events with a higher peak current maxima than that of linear DNA. $N$ refers to the total number of events recorded. (C) The circular plasmid has a significantly higher average current peak maxima than the linearized plasmid, and (D) the linearized plasmid has a significantly longer dwell time than the circular plasmid. The data points in bar charts (C) and (D) correspond to individual events from the current traces. Error bars are \pm SEM. Asterisks indicate $P$-values $(* * * P<0.01)$. The Mann-Whitney test was used for (C) and (D).

time for the linearized DNA $(115 \pm 10 \mu \mathrm{s})$ when compared to that of circular DNA $(101 \pm 10 \mu \mathrm{s})$ (Figure 3D).

Next, we studied whether the observed significant enhancement of the peak amplitude in 50\% (w/v) PEG 8000 could be used to aid the detection of proteins. Various proteins have been studied using solid-state nanopores; ${ }^{6,39-43} \beta$-galactosidase, a $465.4 \mathrm{kDa}$ tetrameric globular protein, has been used as the model protein before and was used as the model protein for this study. ${ }^{44} \beta$-Galactosidase was subjected to size exclusion chromatography to confirm that it was tetrameric (Supporting Figure 8). Different voltages were tested for the translocation of $\beta$-galactosidase, and hereinafter, $-700 \mathrm{mV}$ was chosen and applied for all protein translocation experiments (Supporting Figure 9). The nanopipette was filled with $1 \mu \mathrm{M}$ of $\beta$ galactosidase and immersed into either PBS, PBS with $12.5 \%$ (v/v), 25\% (v/v), or $50 \%(\mathrm{v} / \mathrm{v})$ glycerol, or PBS with $12.5 \%$ $(\mathrm{w} / \mathrm{v}), 25 \%(\mathrm{w} / \mathrm{v}), 50 \%(\mathrm{w} / \mathrm{v})$ PEG 8000 (Figures 4A, 4B, and $4 \mathrm{C}$, Supporting Figure 10). In our system, $\beta$-galactosidase was barely detectable in PBS, or in $12.5-50 \%(\mathrm{v} / \mathrm{v})$ glycerol in PBS, or in $12.5 \%(\mathrm{w} / \mathrm{v})$ or $25 \%(\mathrm{w} / \mathrm{v})$ of PEG 8000 in PBS, with a molecule count fewer than 10 per $60 \mathrm{~s}$ (Figure 4D, Supporting Figure 10). Remarkably, the number of molecules detected exhibited a statistically significant increase in 50\% (w/ v) PEG 8000 of nearly 1000 -fold. The increase in the counted molecules enabled the generation of population scatter and showed a discrete population distribution with the average current maxima of $70 \pm 1 \mathrm{pA}$ and dwell time of $85 \pm 10 \mu \mathrm{s}$. The increase in the counted molecules was concentration dependent (Supporting Figure 11), demonstrating that the peaks detected can be attributed to the protein itself and not to inward PEG 8000 translocations. We observed the highest molecule count at $1 \mu \mathrm{M} \beta$-galactosidase with $893 \pm 30$ proteins per $60 \mathrm{~s}$, followed by $284 \pm 73$ at $0.5 \mu \mathrm{M}$ and $39 \pm 6$ at $0.1 \mu \mathrm{M}$.

Amyloid fibrils are formed when monomeric proteins selfassemble into fibrous protein polymers which have a cross- $\beta$ molecular architecture. ${ }^{45}$ The formation of amyloid fibrils and other filamentous proteins has been studied with nanopores, but complex surface modifications of the nanopore are often required. ${ }^{46-49}$ Having demonstrated that macromolecular crowding improves the nanopore sensitivity for globular proteins, we then investigated whether the detection of $\alpha$ synuclein amyloid fibrils could also be enhanced and whether the length of the amyloid fibrils affected the translocation dynamics. In order to analyze fibrils with distinct length distributions, fragmented and elongated $\alpha$-synuclein amyloid fibrils were generated (see the Methods in the Supporting Information). The length and height distribution of the fibrils were characterized by atomic force microscopy (AFM) (Figure 5A, Supporting Figure 12). The fragmented fibrils had a narrow length distribution with a mean length of $66 \pm 2 \mathrm{~nm}$ and height of $5 \pm 0.1 \mathrm{~nm}$. In contrast, the elongated fibrils had a broad length distribution ranging from 100 to $1600 \mathrm{~nm}$ with a mean length of $442 \pm 12 \mathrm{~nm}$ and height of $6 \pm 0.1 \mathrm{~nm}$ (Figure 5B, Supporting Figure 12).

A nanopipette was filled with a $40 \mu \mathrm{M}$ monomer equivalent concentration of either the fragmented or the elongated fibrils for the translocation experiment. Although the fibril preparations had the same monomer equivalent concentration, due to their increased lengths, the elongated fibrils had a lower particle concentration than the fragmented fibrils. The particle concentration can be calculated from the fibril mass per unit 

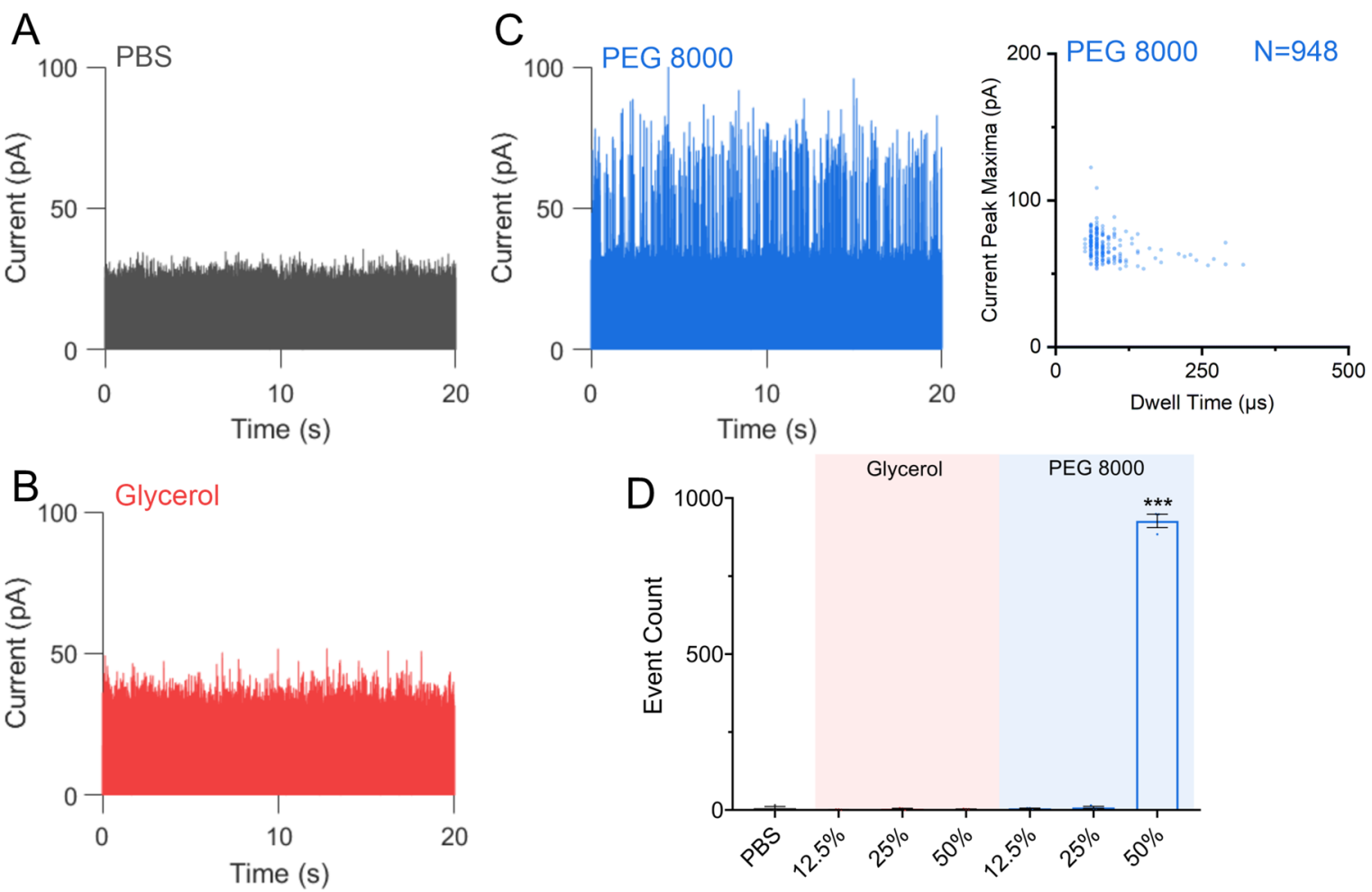

Figure 4. Macromolecular crowding enhances the detection of the globular protein $\beta$-galactosidase. The baseline subtracted current trace for the first $20 \mathrm{~s}$ is shown for electrolyte baths of either (A) PBS, (B) PBS with 50\% (v/v) glycerol, and (C) PBS with 50\% (w/v) PEG 8000. A scatter plot shows the dwell time versus the current peak maxima for each event from the entire $60 \mathrm{~s}$ for $50 \%(\mathrm{w} / \mathrm{v})$ PEG 8000 in PBS bath. $N$ refers to the total number of events recorded. No scatter plots are shown for either PBS or $50 \%(\mathrm{v} / \mathrm{v})$ glycerol PBS due to an insufficient number of events $(<10$ events). (D) Only the 50\% (w/v) PEG 8000 electrolyte bath resulted in a pronounced increase in the number of events. The data points correspond to individual events from the current traces. Error bars are \pm SEM. Asterisks indicate $P$-values $(* * * P<0.01)$. One-way ANOVA was used for (D).

length which has been shown to be $70 \mathrm{kDa}$ per nanometer. ${ }^{50}$ By using the average length of the fibrils, the fragmented fibrils had a 6-fold higher particle concentration $(\sim 150 \mathrm{nM})$ compared with the elongated fibrils $(\sim 25 \mathrm{nM})$. Similar to the results described for $\beta$-galactosidase, very few fragmented and elongated fibrils were detected in PBS $(<10$ in $60 \mathrm{~s})$, but there was a pronounced increase in number of events recorded when the nanopipette was placed into 50\% (w/v) PEG 8000 in PBS (Figures 5C, 5D, and 5E). Single molecule analysis of the translocation of elongated fibrils showed a broad population distribution, potentially due to their inherent heterogeneity in length (Figure 5B). Conversely, the fragmented fibrils, which have a narrower length distribution, had a more homogeneous population compared to the elongated fibrils (Figures $5 \mathrm{C}$ and 5D, Supporting Figure 13). Besides the statistically significant increase in the number of molecules counted in $50 \%(\mathrm{w} / \mathrm{v})$ PEG 8000 when compared to PBS for both fibril populations, there was also a statistically significant increase in the number of molecules counted for the elongated fibrils $(604 \pm 72)$ compared to the fragmented fibrils $(272 \pm 8)$ in $50 \%(\mathrm{w} / \mathrm{v})$ PEG 8000 (Figure 5E). Interestingly, the molecule count for the elongated fibrils was $\sim 3$-fold higher even though the particle concentration was $\sim 6$-fold lower than the fragmented fibrils. This could be due to a subpopulation of the shortest fragmented fibrils not being detected, whereas the elongated fibrils may have been detected more readily because of their larger size. The elongated fibrils also had statistically significant higher average current amplitudes and longer average dwell time $(172 \pm 7 \mathrm{pA}$ and $220 \pm 20 \mu \mathrm{s})$ than the fragmented fibrils
$(106 \pm 7 \mathrm{pA}$ and $83 \pm 10 \mu \mathrm{s}$ ) (Figures 5F and 5G), with the dwell time reflecting the increased average length of the elongated fibrils.

In our system, there was a pronounced effect on the current amplitude when $50 \%(\mathrm{w} / \mathrm{v})$ PEG 8000 was used. To determine whether the size of the PEG polymer influences the observed enhancement of current amplitude, we tested the effect of a $50 \%(\mathrm{w} / \mathrm{v})$ PEG 4000 bath on the detection of a circular plasmid (Supporting Figure 14A) and $\beta$-galactosidase (Supporting Figure 14B). There was no statistically significant difference in the molecule count between PEG 8000 (1063 \pm 24) and PEG $4000(1171 \pm 45)$ for the circular plasmid, but there was a statistically significant lower molecule count for $\beta$ galactosidase in PEG $4000(266 \pm 62)$ than in PEG $8000(893$ $\pm 30)$. The lower molecule count for $\beta$-galactosidase in PEG 4000 may result from the reduced amplification of current amplitude relative to PEG 8000 and due to our stringent threshold level, with translocation events below the threshold level being undetected. This highlights that PEG polymer size is a key determinant for efficient detection of proteins.

In summary, we demonstrate that [i] a crowded, but not viscous, milieu enhances the sensitivity of a solid-state nanopore; [ii] increasing the concentration of the crowding agent markedly increases the current amplitude for DNA, which is in agreement with a previous study utilizing high concentrations of crowding agents; ${ }^{51}$ [iii] the crowding agent enhanced markedly the detection of both globular and fibrillar proteins; and [iv] the increased sensitivity aided the characterization of molecules with different structures and sizes. 
A Fragmented

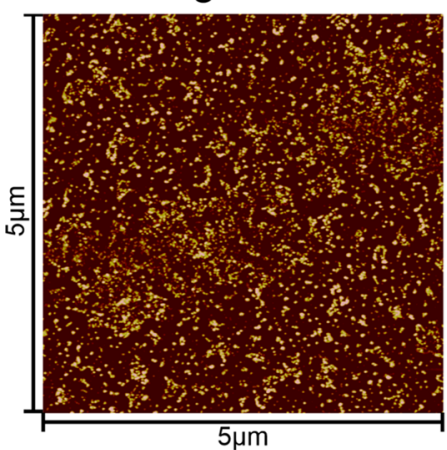

$\mathrm{C}_{400}^{\text {Fragmented }}$

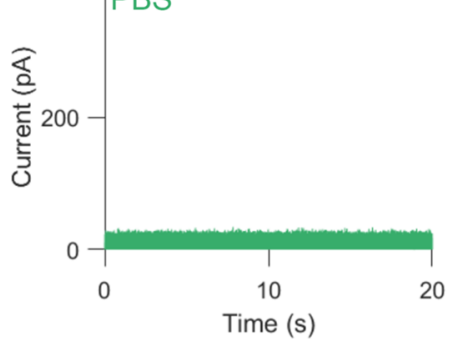

D Fragmented
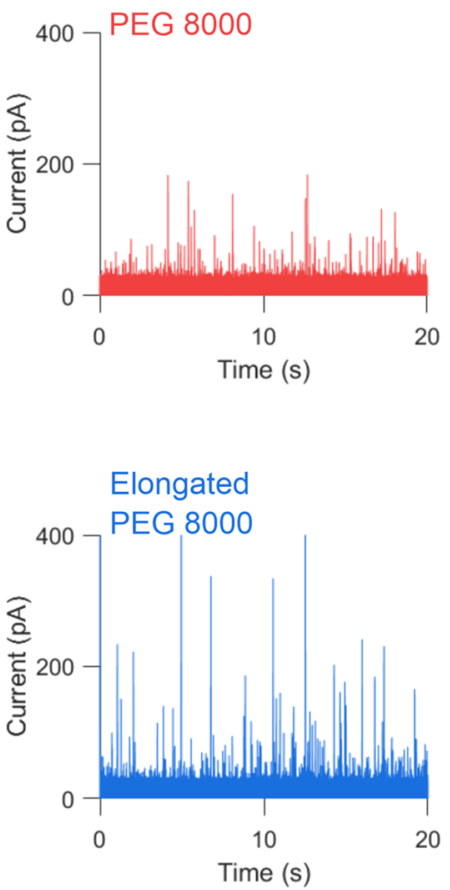

Elongated
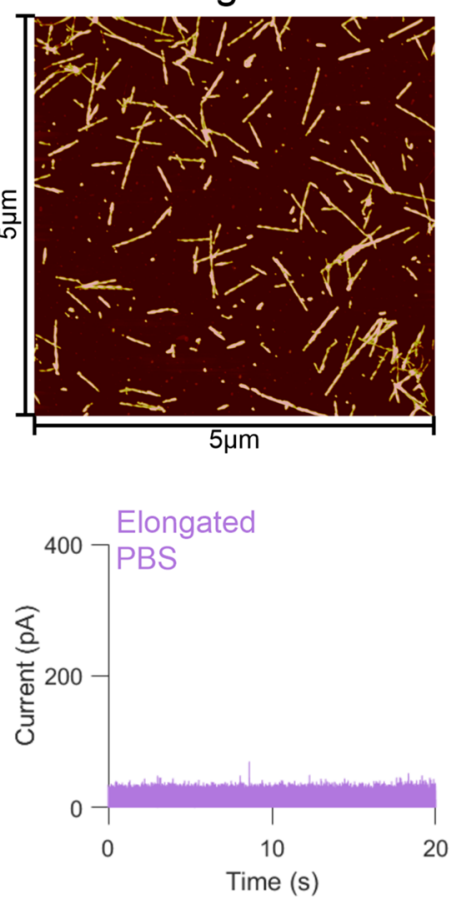

E
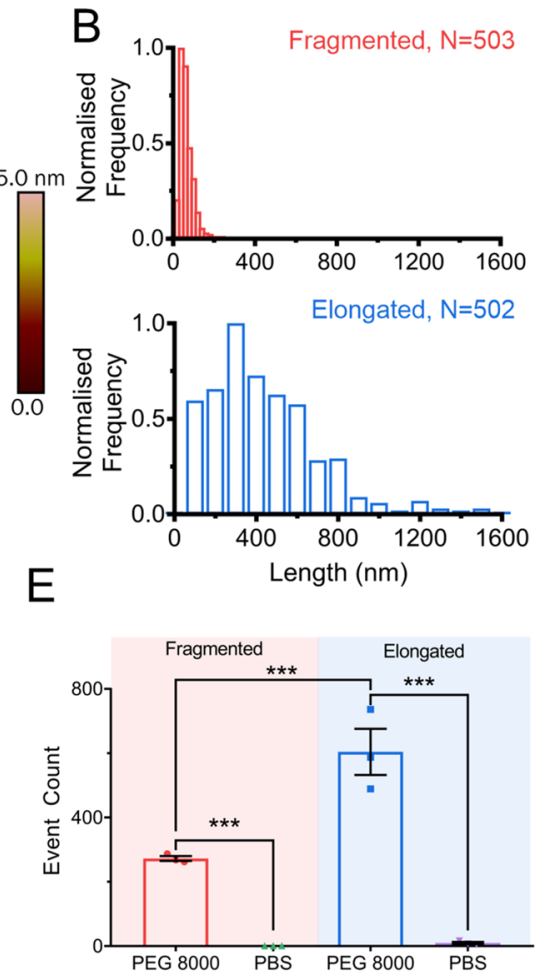

I

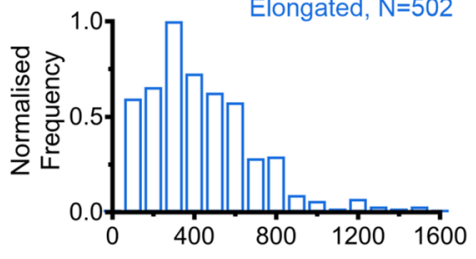

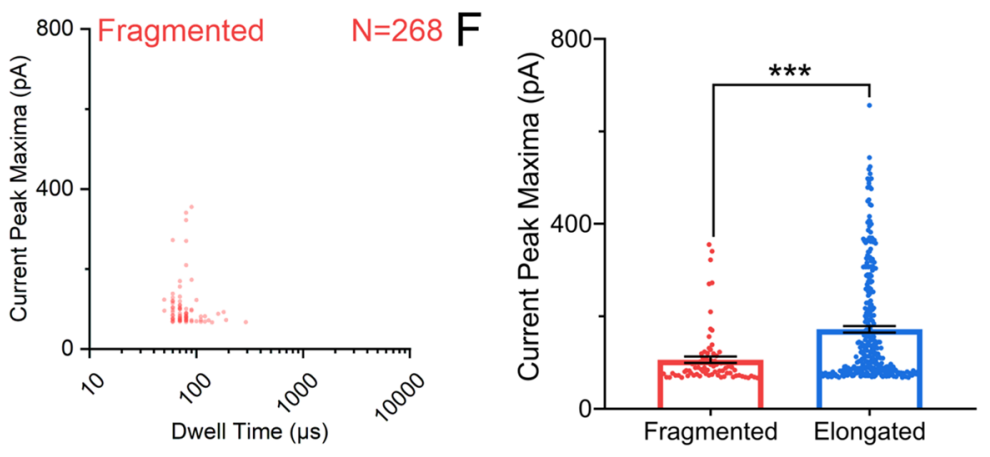
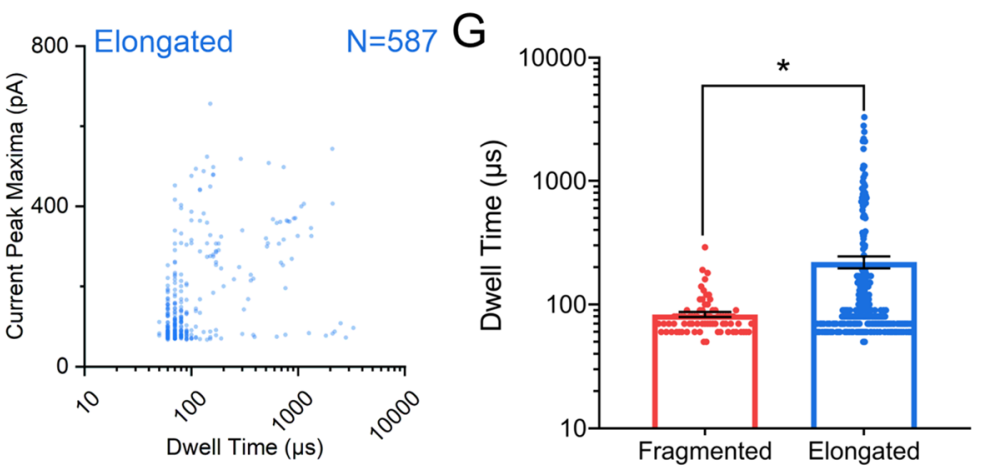

Figure 5. Macromolecular crowding enhances the detection of $\alpha$-synuclein amyloid fibrils. (A) Elongated $\alpha$-synuclein amyloid fibrils were produced by extending fragmented fibrils. Both fibril preparations were imaged by AFM and (B) the length of the fibrils was measured. The mean length of the fragmented fibrils was determined to be $66 \pm 2 \mathrm{~nm}$ with mode at $40 \mathrm{~nm}$ and of the elongated fibrils $442 \pm 12 \mathrm{~nm}$ with mode at 300 $\mathrm{nm}$. The nanopipette was filled with a $40 \mu \mathrm{M}$ monomer equivalent concentration of either fragmented or elongated $\alpha$-synuclein fibrils and immersed into an electrolyte bath. Application of $-700 \mathrm{mV}$ was used to drive the translocation of the fibrils through the nanopore for $60 \mathrm{~s}$. The baseline subtracted current trace for the first $20 \mathrm{~s}$ is shown for electrolyte baths of either (C) PBS or (D) PBS with $50 \%$ (w/v) of the crowding agent PEG 8000. Scatter plots show the dwell time versus the current peak maxima for each event from the entire $60 \mathrm{~s}$ of fibrils. $N$ refers to the total number of events recorded. No scatter plots are shown for PBS due to an insufficient number of events ( $<10$ events). (E) PEG 8000 increased the number of events counted for both the fragmented and elongated fibrils over that of the fibrils in PBS. Moreover, with PEG 8000 the elongated fibrils had a significantly longer dwell time $(\mathrm{F})$ and higher current peak maxima $(\mathrm{G})$ than the fragmented fibrils. Asterisks indicate $P$-values $(* P<$ 0.5 ; $* * P<0.01)$. Error bars are \pm SEM. The data points correspond to individual events from the current traces $(\mathrm{F}$ and $\mathrm{G})$. One-way ANOVA was used for (E), and the Mann-Whitney test was used for (F) and (G). 
However, the most remarkable feature of this method is its simplicity, in that adding a crowding agent to the electrolyte increases the detection efficiency of the nanopore by up to 1000 -fold, without any need for complex surface modification of the nanopore.

In our system we observed that the increasing concentration from $0 \%$ to $50 \%(\mathrm{v} / \mathrm{v})$ of the viscogen glycerol did not affect the molecule count and the translocation dynamics of a circular plasmid and $\beta$-galactosidase. Fologea et al. showed that the addition of $50 \%(\mathrm{v} / \mathrm{v})$ glycerol to $\mathrm{KCl}$ electrolyte caused a pronounced effect on the translocation speed of the DNA through the nanopore by increasing the dwell time from under $200 \mu \mathrm{s}$ to approximately $600 \mu \mathrm{s}$. ${ }^{.}$However, the addition of glycerol to the electrolyte bath reduced the peaks' amplitude, decreasing the signal-to-noise ratio. ${ }^{7,52}$ Thus, despite a predicted increase in dwell time, in our system the reduction of the signal-to-noise ratio in glycerol caused the translocation events to fall below our threshold settings for events calling. Indeed, we observed a gradual reduction in the molecule count for the detection of circular plasmid with increasing concentrations of glycerol. Conversely, a macromolecular crowded environment of PEG 8000 enhanced the detection efficiency by amplifying the translocation events' current amplitudes, thus increasing the signal-to-noise ratio.

How macromolecular crowding enhances the detection of biomolecules by a solid-state nanopore is unclear. An entropydriven model was proposed to explain the observed increase in capture rate, peak amplitude, and dwell time by macromolecular crowding for a $\alpha$-hemolysin $(\alpha \mathrm{HL})$ protein nanopore, ${ }^{51}$ but the same model may not be directly applied to our approach. This is because unlike in Yao et al., where the biomolecules were mixed with the crowded solution and driven to the uncrowded solution, ${ }^{51}$ our method delivers the biomolecules from the uncrowded solution into the crowded solution. Interestingly, the pronounced improvement in the detection of both DNAs and proteins occurred only when the solution was highly crowded at 50\% (w/v) PEG 8000 . This highly crowded environment reversed the negative ion current rectification and may contribute to the observed enhanced detection of biomolecules. The asymmetric concentration gradient between the electrolyte inside the nanopipette and the highly crowded electrolyte bath can affect the electroosmotic flow of the system. ${ }^{53}$ In 2019, Larimi et al. investigated the effect of crowding on the interactions of a polypeptide with a biological nanopore, concluding that crowded conditions resulted in a stronger polypeptide-nanopore interaction. ${ }^{54}$ The enhancement of the capture rate of DNA in biological nanopores by crowding ${ }^{51}$ could be associated with the disruption of electroosmotic flow in the nanopore. Indeed, Yusko et al. demonstrated that the formation of the asymmetric concentration gradient by the addition of various percentages of dimethyl sulfoxide (DMSO) into the electrolyte altered the solution properties as well as the electroosmotic flow in a conical nanopore. ${ }^{23}$ Similarly, Rabinowitz et al. showed that the asymmetric ion concentration gradient affected the ion current rectification of the nanopipette by inducing the formation of nanoscale fluid vortices and nonlinear electroosmotic flow. ${ }^{24}$ Furthermore, studies have shown that the addition of a low concentration of PEG can have an impact on the translocation dynamic of the biomolecules by disrupting the electroosmotic outflow. ${ }^{53,55}$ In our system, the observed enhancement of the sensitivity of the nanopore and the alteration on the translocation dynamics of the biomolecules could be due to the combined effect of the entropy and the modified electroosmotic flow.

In conclusion, we demonstrate that macromolecular crowding improves the detection efficiency of the nanopore for DNA and is particularly effective for the detection of globular and filamentous proteins. We envision that macromolecular crowding could improve the application of solidstate nanopore in single molecule detection and characterization, and our data suggest that single molecule detection in a crowded environment, such as the cytoplasm of a cell, should lead also to improved sensitivity. ${ }^{56}$

\section{ASSOCIATED CONTENT}

\section{sI Supporting Information}

The Supporting Information is available free of charge at https://pubs.acs.org/doi/10.1021/acs.nanolett.0c02246.

Methods and associated supporting figures (PDF)

\section{AUTHOR INFORMATION}

\section{Corresponding Authors}

Eric W. Hewitt - School of Molecular and Cellular Biology and Astbury Centre for Structural Molecular Biology, University of Leeds, Leeds LS2 9JT, U.K.; Email: e.w.hewitt@leeds.ac.uk

Paolo Actis - School of Electronic and Electrical Engineering and Pollard Institute, University of Leeds, Leeds LS2 9JT, U.K.; 이 orcid.org/0000-0002-7146-1854; Email: p.actis@ leeds.ac.uk

\section{Authors}

Chalmers C. Chau - School of Molecular and Cellular Biology and Astbury Centre for Structural Molecular Biology and School of Electronic and Electrical Engineering and Pollard Institute, University of Leeds, Leeds LS2 9JT, U.K.

Sheena E. Radford - School of Molecular and Cellular Biology and Astbury Centre for Structural Molecular Biology, University of Leeds, Leeds LS2 9JT, U.K.

Complete contact information is available at:

https://pubs.acs.org/10.1021/acs.nanolett.0c02246

\section{Funding}

The authors acknowledge funding from the Wellcome Trust (SER) (204963), ERC (SER) (322408), and the University of Leeds (PA).

\section{Notes}

The ionic current trace data associated with this paper are openly available from the University of Leeds Research Data Repository at: https://doi.org/10.5518/841.

The authors declare no competing financial interest.

\section{ACKNOWLEDGMENTS}

We thank Michael Davis and Dr. Matthew P. Jackson for the generation of the fragmented fibrils, Dr. Andrew Lee for helping with the AFM imaging, Dr. Aleksandar P. Ivanov and Prof. Joshua B. Edel for generously providing the MATLAB script used for event analysis in this study, and Mukhil Raveendran and Fabio Marcuccio for event analysis. We thank the members of Radford, Hewitt, and Actis laboratories for helpful discussion. 


\section{ABBREVIATIONS USED}

AFM, atomic force microscopy; $\alpha \mathrm{HL}, \alpha$-hemolysin; DMSO, dimethyl sulfoxide; PBS, phosphate buffered saline; PEG, polyethylene glycol

\section{REFERENCES}

(1) Kasianowicz, J. J.; Brandin, E.; Branton, D.; Deamer, D. W. Characterization of individual polynucleotide molecules using a membrane channel. Proc. Natl. Acad. Sci. U. S. A. 1996, 93 (24), 13770-3.

(2) Muthukumar, M.; Plesa, C.; Dekker, C. Single-molecule sensing with nanopores. Phys. Today 2015, 68 (8), 40-6.

(3) Howorka, S.; Cheley, S.; Bayley, H. Sequence-specific detection of individual DNA strands using engineered nanopores. Nat. Biotechnol. 2001, 19 (7), 636-9.

(4) Loman, N. J.; Watson, M. Successful test launch for nanopore sequencing. Nat. Methods 2015, 12 (4), 303-4.

(5) Jain, M.; Fiddes, I. T.; Miga, K. H.; Olsen, H. E.; Paten, B.; Akeson, M. Improved data analysis for the MinION nanopore sequencer. Nat. Methods 2015, 12 (4), 351-6.

(6) Plesa, C.; Kowalczyk, S. W.; Zinsmeester, R.; Grosberg, A. Y.; Rabin, Y.; Dekker, C. Fast Translocation of Proteins through Solid State Nanopores. Nano Lett. 2013, 13 (2), 658-63.

(7) Fologea, D.; Uplinger, J.; Thomas, B.; McNabb, D. S.; Li, J. Slowing DNA Translocation in a Solid-State Nanopore. Nano Lett. 2005, 5 (9), 1734-7.

(8) Wanunu, M.; Morrison, W.; Rabin, Y.; Grosberg, A. Y.; Meller, A. Electrostatic focusing of unlabelled DNA into nanoscale pores using a salt gradient. Nat. Nanotechnol. 2010, 5 (2), 160-5.

(9) Kowalczyk, S. W.; Wells, D. B.; Aksimentiev, A.; Dekker, C. Slowing down DNA Translocation through a Nanopore in Lithium Chloride. Nano Lett. 2012, 12 (2), 1038-44.

(10) Yusko, E. C.; Johnson, J. M.; Majd, S.; Prangkio, P.; Rollings, R. C.; Li, J.; Yang, J.; Mayer, M. Controlling protein translocation through nanopores with bio-inspired fluid walls. Nat. Nanotechnol. 2011, 6 (4), 253-60.

(11) Iqbal, S. M.; Akin, D.; Bashir, R. Solid-state nanopore channels with DNA selectivity. Nat. Nanotechnol. 2007, 2 (4), 243-8.

(12) Wei, R.; Martin, T. G.; Rant, U.; Dietz, H. DNA Origami Gatekeepers for Solid-State Nanopores. Angew. Chem., Int. Ed. 2012, 51 (20), 4864-7.

(13) Bell, N. A. W.; Engst, C. R.; Ablay, M.; Divitini, G.; Ducati, C.; Liedl, T.; Keyser, U. F. DNA Origami Nanopores. Nano Lett. 2012, 12 (1), 512-7.

(14) Sze, J. Y. Y.; Ivanov, A. P.; Cass, A. E. G.; Edel, J. B. Single molecule multiplexed nanopore protein screening in human serum using aptamer modified DNA carriers. Nat. Commun. 2017, 8 (1), 1552 .

(15) Bell, N. A. W.; Keyser, U. F. Digitally encoded DNA nanostructures for multiplexed, single-molecule protein sensing with nanopores. Nat. Nanotechnol. 2016, 11 (7), 645-51.

(16) Fraccari, R. L.; Carminati, M.; Piantanida, G.; Leontidou, T.; Ferrari, G.; Albrecht, T. High-bandwidth detection of short DNA in nanopipettes. Faraday Discuss. 2016, 193, 459-70.

(17) Shekar, S.; Niedzwiecki, D. J.; Chien, C.-C.; Ong, P.; Fleischer, D. A.; Lin, J.; Rosenstein, J. K.; Drndić, M.; Shepard, K. L. Measurement of DNA Translocation Dynamics in a Solid-State Nanopore at 100 ns Temporal Resolution. Nano Lett. 2016, 16 (7), 4483-9.

(18) Rosenstein, J. K.; Wanunu, M.; Merchant, C. A.; Drndic, M.; Shepard, K. L. Integrated nanopore sensing platform with submicrosecond temporal resolution. Nat. Methods 2012, 9 (5), 487-92.

(19) Benny, P.; Raghunath, M. Making microenvironments: A look into incorporating macromolecular crowding into in vitro experiments, to generate biomimetic microenvironments which are capable of directing cell function for tissue engineering applications. J. Tissue Eng. 2017, 8, 204173141773046.
(20) Raveendran, M.; Lee, A. J.; Wälti, C.; Actis, P. Analysis of 2D DNA Origami with Nanopipettes. ChemElectroChem 2018, 5 (20), 3014-20.

(21) Actis, P.; Mak, A. C.; Pourmand, N. Functionalized nanopipettes: toward label-free, single cell biosensors. Bioanalytical Reviews. 2010, 1 (2-4), 177-85.

(22) Umehara, S.; Pourmand, N.; Webb, C. D.; Davis, R. W.; Yasuda, K.; Karhanek, M. Current Rectification with Poly-l-LysineCoated Quartz Nanopipettes. Nano Lett. 2006, 6 (11), 2486-92.

(23) Yusko, E. C.; An, R.; Mayer, M. Electroosmotic Flow Can Generate Ion Current Rectification in Nano- and Micropores. ACS Nano 2010, 4 (1), 477-87.

(24) Rabinowitz, J.; Edwards, M. A.; Whittier, E.; Jayant, K.; Shepard, K. L. Nanoscale Fluid Vortices and Nonlinear Electroosmotic Flow Drive Ion Current Rectification in the Presence of Concentration Gradients. J. Phys. Chem. A 2019, 123 (38), 8285-93.

(25) Reiner, J. E.; Kasianowicz, J. J.; Nablo, B. J.; Robertson, J. W. F. Theory for polymer analysis using nanopore-based single-molecule mass spectrometry. Proc. Natl. Acad. Sci. U. S. A. 2010, 107 (27), $12080-5$.

(26) Balijepalli, A.; Robertson, J. W. F.; Reiner, J. E.; Kasianowicz, J. J.; Pastor, R. W. Theory of Polymer-Nanopore Interactions Refined Using Molecular Dynamics Simulations. J. Am. Chem. Soc. 2013, 135 (18), 7064-72.

(27) Machado, D. C.; Júnior, J. J. S.; Melo, M. C. A.; Silva, A. M. B.; Fontes, A.; Rodrigues, C. G. Effects of alkali and ammonium ions in the detection of poly(ethyleneglycol) by alpha-hemolysin nanopore sensor. RSC Adv. 2016, 6 (61), 56647-55.

(28) Smeets, R. M. M.; Keyser, U. F.; Krapf, D.; Wu, M.-Y.; Dekker, N. H.; Dekker, C. Salt Dependence of Ion Transport and DNA Translocation through Solid-State Nanopores. Nano Lett. 2006, 6 (1), $89-95$.

(29) Steinbock, L. J.; Lucas, A.; Otto, O.; Keyser, U. F. Voltagedriven transport of ions and DNA through nanocapillaries. Electrophoresis 2012, 33 (23), 3480-7.

(30) Ivanov, A. P.; Actis, P.; Jönsson, P.; Klenerman, D.; Korchev, Y.; Edel, J. B. On-Demand Delivery of Single DNA Molecules Using Nanopipets. ACS Nano 2015, 9 (4), 3587-95.

(31) Chen, K.; Bell, N. A. W.; Kong, J.; Tian, Y.; Keyser, U. F. Direction- and Salt-Dependent Ionic Current Signatures for DNA Sensing with Asymmetric Nanopores. Biophys. J. 2017, 112 (4), 67482.

(32) Aksimentiev, A. Deciphering ionic current signatures of DNA transport through a nanopore. Nanoscale 2010, 2 (4), 468.

(33) Baaken, G.; Ankri, N.; Schuler, A.-K.; Rühe, J.; Behrends, J. C. Nanopore-Based Single-Molecule Mass Spectrometry on a Lipid Membrane Microarray. ACS Nano 2011, 5 (10), 8080-8.

(34) Cao, C.; Ying, Y.-L.; Gu, Z.; Long, Y.-T. Enhanced Resolution of Low Molecular Weight Poly(Ethylene Glycol) in Nanopore Analysis. Anal. Chem. 2014, 86 (24), 11946-50.

(35) Chandrashekaran, S. KpnI restriction endonuclease and methyltransferase exhibit contrasting mode of sequence recognition. Nucleic Acids Res. 2004, 32 (10), 3148-55.

(36) Fologea, D.; Brandin, E.; Uplinger, J.; Branton, D.; Li, J. DNA conformation and base number simultaneously determined in a nanopore. Electrophoresis 2007, 28 (18), 3186-92.

(37) Kumar Sharma, R.; Agrawal, I.; Dai, L.; Doyle, P. S.; Garaj, S. Complex DNA knots detected with a nanopore sensor. Nat. Commun. 2019, 10 (1), 4473.

(38) Steinbock, L. J.; Otto, O.; Chimerel, C.; Gornall, J.; Keyser, U. F. Detecting DNA Folding with Nanocapillaries. Nano Lett. 2010, 10 (7), 2493-7.

(39) Steinbock, L. J.; Krishnan, S.; Bulushev, R. D.; Borgeaud, S.; Blokesch, M.; Feletti, L.; Radenovic, A. Probing the size of proteins with glass nanopores. Nanoscale 2014, 6 (23), 14380-7.

(40) Hu, R.; Rodrigues, J.V.; Waduge, P.; Yamazaki, H.; Cressiot, B.; Chishti, Y.; Makowski, L.; Yu, D.; Shakhnovich, E.; Zhao, Q.; Wanunu, M. Differential Enzyme Flexibility Probed Using Solid-State Nanopores. ACS Nano 2018, 12 (5), 4494-502. 
(41) Waduge, P.; Hu, R.; Bandarkar, P.; Yamazaki, H.; Cressiot, B.; Zhao, Q.; Whitford, P. C.; Wanunu, M. Nanopore-Based Measurements of Protein Size, Fluctuations, and Conformational Changes. ACS Nano 2017, 11 (6), 5706-16.

(42) Giamblanco, N.; Coglitore, D.; Janot, J.-M.; Coulon, P. E.; Charlot, B.; Balme, S. Detection of protein aggregate morphology through single antifouling nanopore. Sens. Actuators, B 2018, 260, 736-45.

(43) Li, W.; Bell, N. A. W.; Hernández-Ainsa, S.; Thacker, V. V.; Thackray, A. M.; Bujdoso, R.; Keyser, U. F. Single Protein Molecule Detection by Glass Nanopores. ACS Nano 2013, 7 (5), 4129-34.

(44) Bartesaghi, A.; Matthies, D.; Banerjee, S.; Merk, A.; Subramaniam, S. Structure of -galactosidase at 3.2-A resolution obtained by cryo-electron microscopy. Proc. Natl. Acad. Sci. U. S. A. 2014, 111 (32), 11709-14.

(45) Iadanza, M. G.; Jackson, M. P.; Hewitt, E. W.; Ranson, N. A.; Radford, S. E. A new era for understanding amyloid structures and disease. Nat. Rev. Mol. Cell Biol. 2018, 19 (12), 755-73.

(46) Giamblanco, N.; Coglitore, D.; Gubbiotti, A.; Ma, T.; Balanzat, E.; Janot, J.-M.; Chinappi, M.; Balme, S. Amyloid Growth, Inhibition, and Real-Time Enzymatic Degradation Revealed with Single Conical Nanopore. Anal. Chem. 2018, 90 (21), 12900-8.

(47) Yusko, E. C.; Prangkio, P.; Sept, D.; Rollings, R. C.; Li, J.; Mayer, M. Single-Particle Characterization of $\mathrm{A} \beta$ Oligomers in Solution. ACS Nano 2012, 6 (7), 5909-19.

(48) Balme, S.; Coulon, P. E.; Lepoitevin, M.; Charlot, B.; Yandrapalli, N.; Favard, C.; Muriaux, D.; Bechelany, M.; Janot, J.M. Influence of Adsorption on Proteins and Amyloid Detection by Silicon Nitride Nanopore. Langmuir 2016, 32 (35), 8916-25.

(49) Wang, X.; Wilkinson, M. D.; Lin, X.; Ren, R.; Willison, K. R.; Ivanov, A. P.; Baum, J.; Edel, J. B. Single-molecule nanopore sensing of actin dynamics and drug binding. Chemical Science. 2020, 11 (4), 970-9.

(50) Iadanza, M. G.; Jackson, M. P.; Radford, S. E.; Ranson, N. A. MpUL-multi: Software for Calculation of Amyloid Fibril Mass per Unit Length from TB-TEM Images. Sci. Rep. 2016, 6 (1), 21078.

(51) Yao, F.; Peng, X.; Su, Z.; Tian, L.; Guo, Y.; Kang, X-f. Crowding-Induced DNA Translocation through a Protein Nanopore. Anal. Chem. 2020, 92 (5), 3827-33.

(52) Li, J.; Talaga, D. S. The distribution of DNA translocation times in solid-state nanopores. J. Phys.: Condens. Matter 2010, 22 (45), 454129.

(53) Wang, V.; Ermann, N.; Keyser, U. F. Current Enhancement in Solid-State Nanopores Depends on Three-Dimensional DNA Structure. Nano Lett. 2019, 19 (8), 5661-6.

(54) Larimi, M. G.; Mayse, L. A.; Movileanu, L. Interactions of a Polypeptide with a Protein Nanopore Under Crowding Conditions. ACS Nano 2019, 13 (4), 4469-77.

(55) Ermann, N.; Hanikel, N.; Wang, V.; Chen, K.; Weckman, N. E.; Keyser, U. F. Promoting single-file DNA translocations through nanopores using electro-osmotic flow. J. Chem. Phys. 2018, 149 (16), 163311.

(56) Pan, R.; Hu, K.; Jia, R.; Rotenberg, S. A.; Jiang, D.; Mirkin, M. V. Resistive-Pulse Sensing Inside Single Living Cells. J. Am. Chem. Soc. 2020, 142 (12), 5778-84. 